\title{
US city-size distribution and space
}

\section{Rafael González-Val}

To cite this article: Rafael González-Val (2019): US city-size distribution and space, Spatial Economic Analysis, DOI: 10.1080/17421772.2019.1572917

To link to this article: https://doi.org/10.1080/17421772.2019.1572917

\section{Published online: 13 Feb 2019.}

Submit your article to this journal ๔

\section{View Crossmark data $\nearrow$}




\title{
US city-size distribution and space
}

\author{
Rafael González-Val
}

\begin{abstract}
This paper focuses on spatial city-size distribution in the United States. It proposes a new distance-based approach to analyze the influence of distance on the city-size distribution parameter by considering the Pareto distribution and using data from different definitions of US cities in 2010. Considering all possible combinations of cities within a 300-mile radius, the results indicate that the Pareto distribution cannot be rejected in most cases regardless of city size. Placebo regressions validate the results, thereby confirming the significant effect of geography on the Pareto exponent.
\end{abstract}

\section{KEYWORDS}

space, city-size distribution, distance-based approach, Pareto distribution, Zipf's law

JEL C12, C14, R11, R12

HISTORY Received 27 March 2018; in revised form 14 December 2018

\section{INTRODUCTION}

In 1913, Auerbach found a striking empirical regularity that establishes a linear and stable relationship between city size and rank - it has fascinated researchers from many fields (e.g., economics, statistics, physics and geography) ever since. In statistical terms, this relationship means that city-size distribution can be fitted well with a Pareto distribution, which is also known as a power law. Some decades later, this empirical regularity became known as Zipfs law (Zipf, 1949), although Zipfs law is simply a particular case of that linear relationship where the parameter of the Pareto distribution is equal to 1 , which means that, when ordered from largest to smallest, the size of the second-largest city in a country is half that of the first, the size of the third is one-third of the first, etc. Over the years, numerous studies have tested the validity of this law for many different countries (see the surveys by Cheshire, 1999; Nitsch, 2005; and, more recently, Cottineau, 2017).

Although interest in city-size distributions and Zipf's law has fluctuated over time, in the last few decades there has been a revival of interest among urban economists, especially since Krugman (1996a) highlight the 'mystery of urban hierarchy'. In a fundamental contribution, Krugman (1996b) uses data from metropolitan areas from the Statistical Abstract of the United States (135 cities) and concludes that in 1991 the Pareto's exponent was exactly equal to 1.005. This finding provides evidence supporting Zipfs law at that time in the United States. Zipfs law provides a simple but accurate representation of city-size distribution and, therefore, some theoretical models with different economic foundations have been proposed to explain the law: productivity 
or technology shocks (Duranton, 2007; Rossi-Hansberg \& Wright, 2007) or local random amenity shocks (Gabaix, 1999). These models justify Zipf's law analytically, associate it directly with an equilibrium situation and connect it to proportionate city growth (Gibrat's law, another wellknown empirical regularity that postulates that the growth rates of cities tend to be independent of their initial sizes). In the theoretical literature, Zipfs law was viewed as a reflection of a steadystate situation.

However, things changed after the publication of Eeckhout (2004). Traditionally due to data limitations, most of the studies have considered only the largest cities. However, Eeckhout demonstrated the statistical importance of considering both large and small cities. Truncated samples lead to biased results, and city definition (administrative cities versus metro areas) also plays a key role in the final results (Rosen \& Resnick, 1980). However, in a larger blow to Zipf's law, Eeckhout (2004) concludes that city-size distribution is actually lognormal rather than Pareto. Since then, most studies have considered untruncated data (Giesen, Zimmermann, \& Suedekum, 2010; González-Val, Ramos, Sanz-Gracia, \& Vera-Cabello, 2015; Ioannides \& Skouras, 2013), but the lognormal distribution soon was replaced by other more convoluted distributions that provide a better fit to the actual data: the $q$-exponential distribution (Malacarne, Mendes, \& Lenzi, 2001; Soo, 2007), the double Pareto lognormal distribution (Giesen et al., 2010; Giesen \& Südekum, 2014; Reed, 2002), or the distribution function by Ioannides and Skouras (2013) that switches between a lognormal and a power distribution.

Most of these new distributions combine linear and non-linear functions, separating the body of the distribution from the upper-tail behaviour. The reason is that the largest cities encompass most of the population of a country, and the behaviour of the upper-tail distribution can be different from that of the entire distribution. In fact, the largest cities follow a Pareto distribution in many cases (Levy, 2009). As Ioannides and Skouras (2013) pointed out, 'most cities obey a lognormal; but the upper-tail and therefore most of the population obeys a Pareto law' (p. 18, emphasis in original).

Therefore, if the Pareto distribution is still valid but only for the upper-tail distribution (i.e., the largest cities), it is possible to reconcile the traditional literature focused on small data sets of large cities with the last empirical studies considering all cities with no size restrictions and the theoretical models considering Zipf's law as the benchmark for the distribution of city sizes. However, this solution is unsatisfactory for two main reasons.

First, urban theoretical models should attempt to explain city sizes and urban systems without imposing any size restriction. It is true that the Pareto distribution provides a simple theoretical specification to include in an analytical framework, but if models are restricted to studying only the largest cities at the upper tail of the distribution, where Zipf's law holds, we are excluding the majority of cities, which actually are small and medium size, from the analysis. It is not easy to justify from a theoretical or empirical point of view the exclusion of most cities, particularly when there is empirical evidence indicating that the lower tail of the distribution, the smallest cities, are also Pareto distributed (Giesen et al., 2010; Giesen \& Südekum, 2014; Luckstead \& Devadoss, 2017; Reed, 2001, 2002).

Second, a Pareto distribution can be fit to a wide range of phenomena: the distribution of the number of times that different words appear in a book (Zipf, 1949), the losses caused by floods (Pisarenko, 1998) and the intensity of wars or forest fires (Roberts \& Turcotte, 1998), for example. However, the city-size distribution case is different because there is a spatial dependence among the elements of the distribution; cities are connected through migratory flows. An essential assumption in urban models to obtaining spatial equilibrium is free migration across cities. Therefore, there is a relationship between the population of one city and the populations of nearby cities. Nevertheless, the upper tail of the distribution contains large cities that typically are very far away from one another. For instance, the bilateral physical distance between New York, the largest city in the United States, and Los Angeles, the second largest city, is more than 2400 miles. If we consider the 10 largest cities in the United States in 2010, the average physical distance between these 
cities is greater than 1000 miles. Therefore, on average, there is a significant distance between the largest cities. Is it therefore possible that migration could be significant between these cities? ${ }^{1}$

Rauch (2014) answers this question by using the 2000 US Census to collect data pertaining to the distances that people move. He creates bins of size $100 \mathrm{~km}$ (approximately 62 miles) and concludes that the large majority of people (over $68 \%$ of observations) fall into the bin with a distance between 0 and $100 \mathrm{~km}$. This finding suggesting that the majority of US citizens live near their birthplace. Rauch also finds that the relationship between the number of people and the distance between home and place of birth decreases with distance by estimating a standard gravity equation.

Therefore, there can be migrations between the largest cities even if they are far from one another. However, these migrations are not significant because most people do not move so far. Therefore, it is not clear whether we can use a spatial equilibrium model to explain the distant largest cities as a whole, and what means that the Pareto distribution (and Zipfs law), which represents the steady city-size distribution in many theoretical models (Duranton, 2007; Gabaix, 1999; Rossi-Hansberg \& Wright, 2007), holds for the largest cities because they are almost independent elements. This situation implies that the largest cities are the centres of different urban systems. There are different theories that can explain a hierarchical system of cities with a multiplicity of equilibria, from the classical theory of the central place by Christaller and Lösch and von Thünen's model to more recent models that update these theories, including modern agglomeration economies (e.g., Fujita, Krugman, \& Mori, 1999; Hsu, 2012). However, the empirical literature on city-size distribution typically omits this spatial issue. As a result, the interpretation of results has been reduced to identify the Pareto upper tail, irrespective of whether there is any meaningful relationship between the largest cities. A few exceptions are Dobkins and Ioannides (2001) and Ioannides and Overman (2004).

Other authors also argue for the need to focus on the regional level rather than the overall citysize distribution for the whole country (although both can be related). Gabaix (1999) shows that if urban growth in all regions follows Gibrat's law, we should observe the Zipfian upper-tail distribution on both the regional and national levels. Giesen and Südekum (2011) test this hypothesis for the German case, finding that Zipfs law is satisfied not only for Germany's national urban hierarchy but also in single German regions. Lalanne (2014) studies the hierarchical structure of the Canadian urban system; splitting the Canadian territory into two parts (east and west) allows her to identify different dynamics that were not observable when studying the country as a whole. Finally, Hsu, Mori, and Smith (2014) analyze the size distribution of US core-based statistical areas (CBSAs) using subsets of cities. These authors find that spatial partitions of cities based on geographical proximity are significantly more consistent with the Pareto distribution than are random partitions.

In this study, we develop a new methodology to analyze how city-size distribution changes over space. This paper considers all of the possible combinations of cities within a 300-mile radius. The next section presents the database used. The third section introduces a new distance-based approach to study the influence of distance on the city-size distribution parameter. The fourth section checks the significance of that relationship with some robustness checks, including placebo regressions. The fifth section discusses the results. The sixth section concludes.

\section{DATA}

There are many definitions of cities, but the two common alternatives in the literature are the metropolitan areas and the administratively defined cities (legal cities). Here we consider three different city definitions: places, urban areas and CBSAs. Table 1 lists the descriptive statistics. The data derive from the 2010 US decennial census. Geographical coordinates (latitude and longitude) necessary to compute the bilateral distances between cities were obtained from the 2010 
Table 1. Descriptive statistics.

\begin{tabular}{lrrrrrr}
\hline City definition & Cities & \multicolumn{1}{c}{$\begin{array}{c}\text { Mean } \\
\text { size }\end{array}$} & $\begin{array}{c}\text { Standard } \\
\text { deviation }\end{array}$ & Minimum & Maximum & $\begin{array}{c}\text { Percentage of } \\
\text { the US } \\
\text { population }\end{array}$ \\
\hline Places & 28,738 & 7880.2 & $66,192.9$ & 1 & $8,175,133$ & $73.3 \%$ \\
Urban areas & 3592 & $70,363.7$ & $495,447.5$ & 2500 & $18,351,295$ & $81.9 \%$ \\
Core-based & 929 & $310,836.9$ & $1,056,227.6$ & 13,477 & $19,567,410$ & $93.9 \%$ \\
statistical areas & & & & & & \\
(CBSAs) & & & & & & \\
\hline
\end{tabular}

Source: US Census 2010.

Census US Gazetteer files. ${ }^{2}$ This same data set is used by González-Val (2019a) to study the spatial distribution of US cities; therefore, the exposition here follows closely both the geographical terms and concepts of the US Census Bureau and González-Val's data description.

The generic denomination 'places' has included all incorporated and unincorporated places since the 2000 Census. According to US Census Bureau guidelines, ${ }^{3}$ the generic term 'incorporated place' designates a type of governmental unit incorporated under state law, 'established to provide governmental functions for a concentration of people'. An incorporated place usually is a city, town, village or borough, but can have other legal descriptions. On the other hand, there are 'unincorporated places' (which were renamed census-designated places in the 1980 Census), which designate 'settled concentrations of population that are identifiable by name but are not legally incorporated under the laws of the state in which they are located'. Thus, the difference between incorporated and unincorporated places is merely political and/or administrative in most cases. In the last years these places have been used in empirical studies of American citysize distribution (Eeckhout, 2004; Giesen et al., 2010; González-Val, 2010; Levy, 2009), and their primary advantage is that this city definition does not impose any truncation point (populations range from 1 to $8,175,133$ inhabitants).

'Urban area' is the generic term for urbanized areas and urban clusters. As the US Census Bureau indicates, ${ }^{4}$ 'urbanized areas consist of a densely developed area that contains 50,000 or more people', while 'urban clusters consist of a densely developed area that has a least 2,500 people but fewer than 50,000 people'. Therefore, a minimum size restriction of 2500 inhabitants is imposed (Table 1). The US Census Bureau classifies all territory and population located within an urbanized area or urban cluster as urban and all areas outside as rural. Previous empirical studies based on this definition of urban areas include Garmestani, Allen, and Bessey (2005) and Garmestani, Allen, and Gallagher (2008). Moreover, urban areas are used as the cores for which CBSAs are defined.

Finally, CBSAs are defined by the US Census Bureau ${ }^{5}$ as:

the county or counties or equivalent entities associated with at least one core (urbanized area or urban cluster) of at least 10,000 population, plus adjacent counties having a high degree of social and economic integration with the core as measured through commuting ties with the counties associated with the core.

The term CBSAs includes both metropolitan and micropolitan statistical areas. The difference between them is the classification of the core as urbanized area or urban cluster. Following the US Census Bureau definitions, 'metropolitan statistical areas are CBSAs associated with at least one urbanized area that has a population of at least 50,000' and 'micropolitan statistical areas are CBSAs associated with at least one urban cluster that has a population of at least 10,000 but less than 50,000' people. 
Note that the city definitions used here are nested; most places are included in urban areas, and most urban areas and places are located inside CBSAs. For research purposes, any of these spatial units have pros and cons. The three samples include most of the population of the country $(73.3 \%$ of the total US population lives in places, $81.9 \%$ is located in urban areas and $93.9 \%$ is included in CBSAs). Places are administratively defined cities (legal cities), and their boundaries make no economic sense. However, some factors, such as human capital spillovers, are believed to operate at a very local level (Eeckhout, 2004). Urban areas represent urban agglomerations from which rural locations are excluded. Moreover, CBSAs are more natural economic units; they cover huge areas that are meant to capture labour markets. CBSAs have economic meaning because they include the core area with a population nucleus together with adjacent communities with a high degree of economic and social integration with that core. Nevertheless, Eeckhout (2004) demonstrated the statistical importance of considering the whole sample. Eeckhout recommends the use of places (untruncated data) rather than metro areas (urban areas or CBSAs) because if any truncation point is imposed, the estimates of the Pareto exponent may be biased.

\section{THE SPATIAL CITY-SIZE DISTRIBUTION}

This paper studies how city-size distribution changes over distance. However, the exercise is not a spatial econometrics one. City-size distribution can be estimated using spatial econometrics techniques to account for spatial dependence. Le Gallo and Chasco (2008) consider Spanish urban areas from 1900 to 2001 to estimate Zipfs law using a spatial seemingly unrelated regressions model. The present approach is different: space is introduced into the methodology through the selection of geographical samples of cities based on distances.

Therefore, the first step is to define the geographical samples of neighbouring cities. Different criteria can be used to select the samples. For instance, Hsu et al. (2014) consider a fixed number of samples (regions) using geographical (travel distance between cities) and economic (trade linkages) criteria. Berry and Okulicz-Kozaryn (2012) use a labour market criterion, based on commuting time to jobs located in urban cores. Therefore, depending on the criterion, one obtains a concrete set of subsystems with particular groups of neighbouring cities. Because there are many alternative criteria (based on economic, social or geographical factors) that could give rise to different groups of cities, this paper follows an agnostic view: it considers all the possible combinations of cities within a 300-mile radius based on physical geographical distances. The choice of this threshold is based on a conservative criterion; although Rauch (2014) concludes that most of people in the United States (over 68\% of observations) live near their place of birth (within $100 \mathrm{~km}$ ) and therefore the extent of spatial interactions between cities is reduced to this short distance, this paper considers a higher threshold of 300 miles, which is roughly one-third of the median distance between all pairs of cities (848 miles for places, 857 for urban areas and 820 for CBSAs, to be precise). ${ }^{6}$

Bilateral distances between all cities are calculated using the haversine distance measure. ${ }^{7} \mathrm{Cir}-$ cles of radius $r=15,20, \ldots, 300$ miles are then drawn around the geographical centroid of each city's coordinates, starting from a minimum distance of 15 miles, adding 5 miles each time; ${ }^{8}$ in the case of CBSAs we start the procedure at 50 miles because they are large spatial units encompassing huge areas and therefore for short distances there are very few units. A total of 58 different geographical samples are obtained for each city for places and urban areas and 51 different geographical samples in the case of CBSAs. This exercise is repeated for all cities, considering both places and urban areas. A total of 1,666,804 (28,738 × 58), 208,336 $(3592 \times 58)$ and 47,379 $(929 \times 51)$ geographical samples for places, urban areas and CBSAs, respectively, are recovered. Note that, within these geographical samples, all cities with no size restriction are considered. Obviously, the number of cities included within the circles also increases as distance increases; the fourth section below explicitly analyzes the relationship between geographical distance and sample size. 
Finally, in some cases samples are repeated (different circles include exactly the same cities) or are single-city samples. These issues will be dealt with below.

Once we defined the geographical samples, we examined the behaviour of city-size distribution from this spatial perspective. As noted above, the Pareto distribution is the benchmark in both the theoretical and the empirical literature on city-size distribution.

Let $S$ denote the city size (measured by the population); if $S$ is distributed according to a power law, also known as a Pareto distribution, ${ }^{9}$ the density function is:

$$
p(S)=\frac{a-1}{\underline{S}}(S / \underline{S})^{-a} \quad \forall S \geq \underline{S}
$$

and the complementary cumulative density function $P(S)$ is:

$$
P(S)=(S / \underline{S})^{-a+1} \quad \forall S \geq \underline{S},
$$

where $a>0$ is the Pareto exponent (or the scaling parameter); and $\underline{S}$ is the population of the city at the truncation point. The Pareto distribution is the typical distribution without a characteristic scale; urban systems are complex systems for which we cannot determine the characteristic scales in many cases (Chen \& Zhou, 2008). Therefore, some authors favour quantitative analysis based on scaling instead of quantitative analysis based on characteristic scales, and the solution to an equation of scaling relation is always a power law.

It is easy to obtain the expression $R=A \cdot S^{-a}$, which relates the empirically observed rank $R$ ( 1 for the largest city, 2 for the second largest, and so on) to city size. This expression has been used extensively in urban economics to study city-size distribution (Cheshire, 1999; Gabaix \& Ioannides, 2004).

First, we tested whether this distribution provides an acceptable fit to the geographical samples of cities. For each geographical sample, we used the statistical test for goodness of fit proposed by Clauset, Shalizi, and Newman (2009), ${ }^{10}$ and recently used by González-Val (2019b) to analyze the evolution of the European urban system in the period 1300-1800. The test is based on a measurement of the 'distance' between the empirical distribution of the data and the hypothesized Pareto distribution. This distance is compared with the distance measurements for comparable synthetic data sets drawn from the hypothesized Pareto distribution, and we defined the $p$-value as the fraction of the synthetic distances larger than the empirical distance. This semi-parametric bootstrap approach is based on the iterative calculation of the Kolmogorov-Smirnov (KS) statistic for 100 bootstrap data set replications. ${ }^{11}$ The Pareto exponent is estimated for each geographical sample of cities using the maximum likelihood (ML) estimator, and then the KS statistic is computed for the data and the fitted model. ${ }^{12}$ Single-city samples are excluded. The test samples from the observed data and checks how often the resulting synthetic distribution fit the actual data as poorly as the ML-estimated power law. Therefore, the null hypothesis is the power law behaviour of the original sample. Nevertheless, this test has an unusual interpretation because, regardless of the true distribution from which the data were drawn, we can always fit a power law. Clauset et al. (2009) recommend the conservative choice that the power law is ruled out if $p<0.1$, that is, if there is a probability of 1 in 10 or less that we would obtain data merely by chance that agree as poorly with the model as the data that we have. Therefore, this procedure only allows it to be concluded whether the power law achieves a plausible fit with the data.

Figure 1 shows the result of the Pareto test by distance. For each distance, the graphs represent the fraction of $p<0.1^{13}$ divided by the total number of tests carried out at that distance. ${ }^{14}$ Regarding places (Figure 1(a)), the percentage of rejections of the Pareto distribution, clearly increases with distance, but it is always below $40 \%$, even for the longest distance considered. One explanation for the increasing number of rejections is as follows: the power law can be replaced by another type of distribution function such as a lognormal distribution when we consider untruncated data and when distance and sample size increase. ${ }^{15}$ 


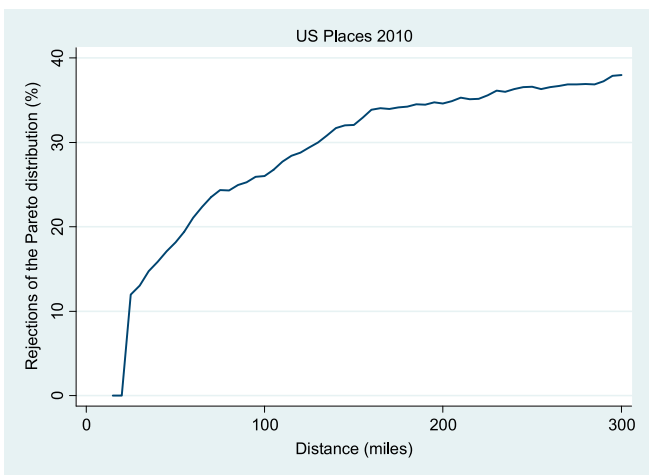

(a) Places

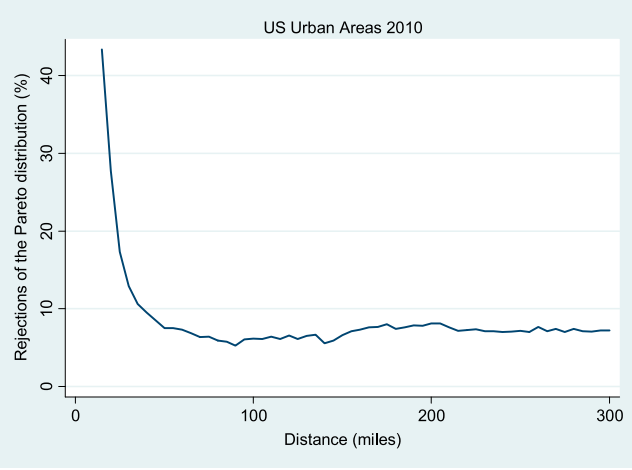

(b) Urban areas

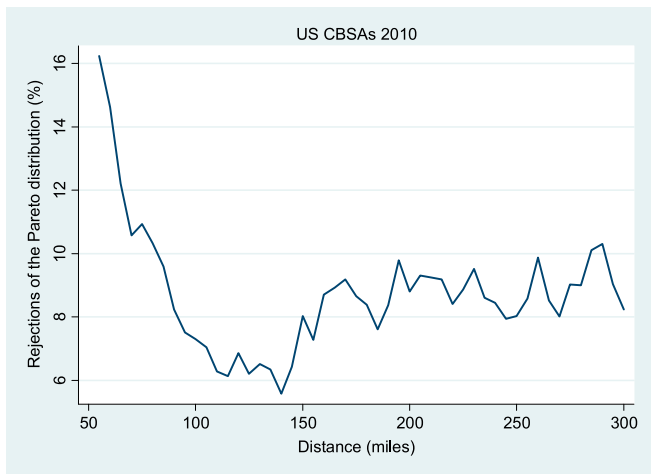

(c) CBSAs

Figure 1. Pareto distribution test over space.

Note: The percentage of rejections of the goodness-of-fit test proposed by Clauset et al. (2009) at the $10 \%$ level.

The results for urban areas and CBSAs are similar (Figure 1(b, c)). For small sample sizes at short distances, the percentage of power law rejections is high but less than $50 \%$. As distance increases, the rejection rate decreases to a rather constant value less than $10 \%$. This situation suggests that the Pareto distribution is a plausible approximation for the real behaviour of the data in the geographical samples in all cases, for any distance, and for the three definitions of city we adopt. Recall that we do not impose any size restriction; therefore, nearby cities are Pareto distributed regardless of the size of the cities included in the samples. Most of the possible combinations of neighbouring cities, for which economic interactions and migratory flows are significant, are Pareto distributed.

Once we conclude that the Pareto distribution is an acceptable description of city sizes, we proceed to estimate the Pareto exponent. Although previously we have estimated the parameter by ML to run the goodness-of-fit test, now we apply Gabaix and Ibragimov's Rank-1/2 estimator. The reason for this choice is that this estimator performs better with small samples. However, when the sample size is large, differences between estimators are reduced (González-Val, 2012). Moreover, Gabaix and Ibragimov (2011) suggest that their estimator produces more robust results than the ML estimator when data deviate from a power law distribution.

Taking natural logarithms from the expression $R=A \cdot S^{-a}$, we obtain the linear specification that is typically estimated:

$$
\ln R=b-a \ln S+\xi
$$


where $\xi$ is the error term; and $b$ and $a$ are parameters that characterize the distribution. Gabaix and Ibragimov propose specifying equation (1) by subtracting $1 / 2$ from the rank to obtain an unbiased estimation of $a$ :

$$
\ln \left(R-\frac{1}{2}\right)=b-a \ln S+\varepsilon
$$

The larger the coefficient $\hat{a}$, the more homogeneous are the city sizes. Similarly, a small coefficient $(<1)$ indicates a heavy-tailed distribution. Zipf's law is an empirical regularity that appears when the Pareto exponent of the distribution is equal to unity $(a=1)$.

Equation (2) is estimated iteratively by ordinary least squares (OLS) for all the geographical samples by distance starting from every city. In other words, we obtain 58 different estimates (51 for CBSAs) of the Pareto exponent for each city. After running all the regressions, we obtain $1,665,962$ Pareto exponent-distance pairs for places, 204,959 in the case of urban areas and 45,912 for CBSAs. Single-city samples are excluded. ${ }^{16}$ Next, to summarize all these point estimates, we conduct a non-parametric estimation of the relationship between distance and the estimated Pareto exponents using local polynomial smoothing. The local polynomial smoother fits the Pareto exponent to a polynomial form of distance via locally weighted least squares, and a Gaussian kernel function is used to calculate the locally weighted polynomial regression. ${ }^{17}$ Figure 2 shows the results, including the $95 \%$ confidence intervals. The results are similar for the three city definitions: as distance increases, the Pareto exponent decreases. The decreasing Pareto exponent converges to the value estimated for the entire sample of cities, which is represented by the horizontal line in Figure 2. A possible explanation for this convergence is that, as distance increases, so does the number of cities within the samples. This situation decreases the coefficient (Eeckhout, 2004). The fourth section below discusses the placebo regressions run to test whether sample size is the only factor driving the results. Finally, the estimated coefficients of urban areas and CBSAs tend to be higher than those of places because of the different definition of cities (González-Val, 2012). Empirical research has established that the city size data are typically well described by a power law with an exponent between 0.8 and 1.2 (Gabaix, 2009). In the case of urban areas and CBSAs, the average estimated exponent is between 0.8 and 1.2 for all distances beyond 30 and 75 miles, respectively. Moreover, for short distances (50-75 miles for urban areas and 75-80 miles in the case of CBSAs), an exponent of 1 falls within the confidence bands. Therefore, we cannot reject Zipfs law for those geographical samples at those distances.

\section{ROBUSTNESS CHECKS}

This section carries out some robustness checks. Previous results have indicated that the Pareto distribution is an acceptable approximation for the real behaviour of the data in the geographical samples, for any distance and for the three city definitions. Moreover, nearby cities are Pareto distributed regardless of the size of the cities included in the samples because we do not impose any size restrictions.

\section{Repeated estimations}

In some cases, some of the geographical samples may be repeated. Recall that we draw circles of different radii from 0 to 300 miles starting from each city to consider all the possible combinations of cities. Therefore, if the core cities of two different circles are close, the geographical samples may be similar or even identical. Many repeated observations could be driving these results. To check whether this situation was a problem, we repeated the analysis considering only geographical samples with a core city of more than 100,000 inhabitants. The largest places tend to be dispersed geographically; if we consider only the geographical samples with a large core city, we should avoid replicated samples. 


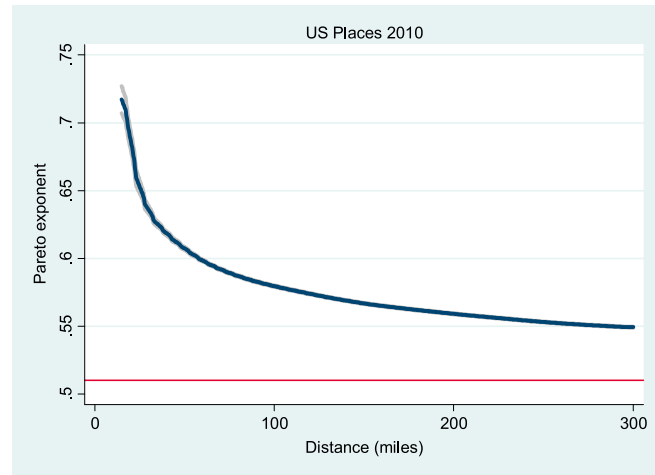

(a) Places

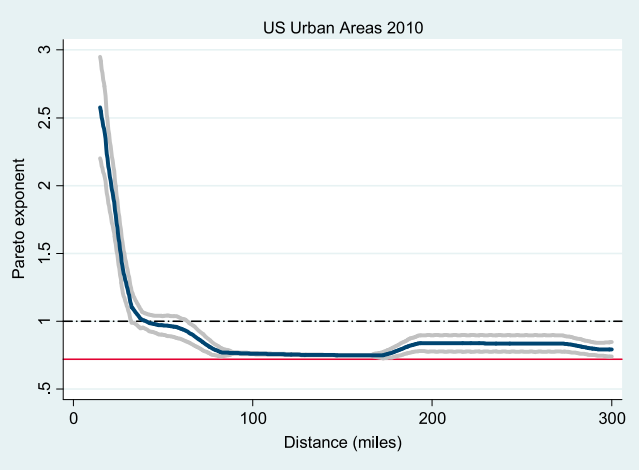

(b) Urban areas

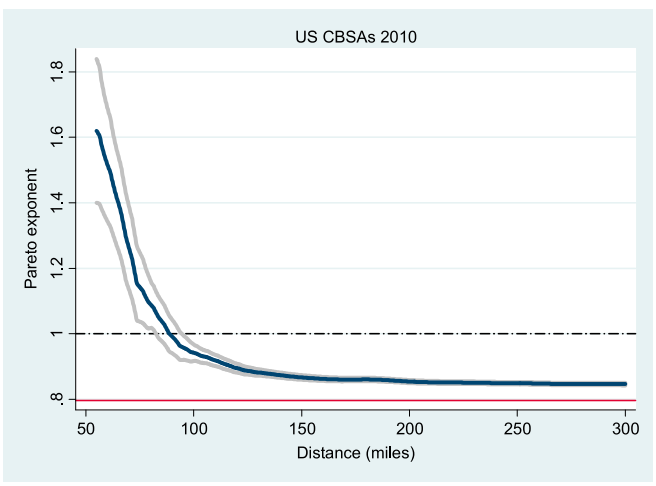

(c) CBSAs

Figure 2. Pareto exponent by distance.

Note: The non-parametric relationship between distance and the estimated Pareto exponents including the 95\% confidence intervals, based on 1,665,962 (a), 204,959 (b) and 45,912 (c) Pareto exponentdistance pairs. The horizontal line indicates the estimated Pareto exponent for the entire sample of cities.

Figure 3 shows the results of the Pareto goodness-of-fit test. Figure 3(a-c) displays a similar evolution of the percentage of rejections with distance to that shown in Figure $1(\mathrm{a}-\mathrm{c})$ when all the geographical samples are considered. ${ }^{18}$ The only difference is that now the percentage of rejections in the case of places is slightly higher, especially for the largest distances, when it reaches $51 \%$. Nevertheless, we can still argue that the Pareto distribution is a plausible fit to the city-size distribution for places for most distances. For urban areas and CBSAs, the percentage of rejections remains below $10 \%$ for most distances.

The sample selection of this robustness check reduces the number of point estimates of the Pareto exponent; now we obtain 16,237 Pareto exponent-distance pairs for places, 17,034 for urban areas and 18,530 in the case of CBSAs. Figure 4 shows the non-parametric relationship between the Pareto exponent and distance, which is still decreasing in the three cases. In the case of urban areas, the Pareto exponent strongly decreases from 0 to 50 miles and then starts to increase slowly as it approaches the estimated value for the entire sample (the horizontal line in Figure 4) from below.

\section{Placebo regressions}

Consider geographical samples that represent all the possible combinations of cities within a 300mile radius. Each geographical sample includes a particular number of cities; there are 1,666,804, 


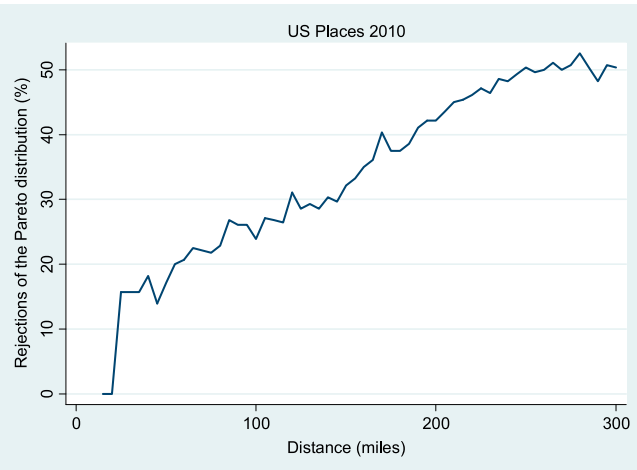

(a) Places

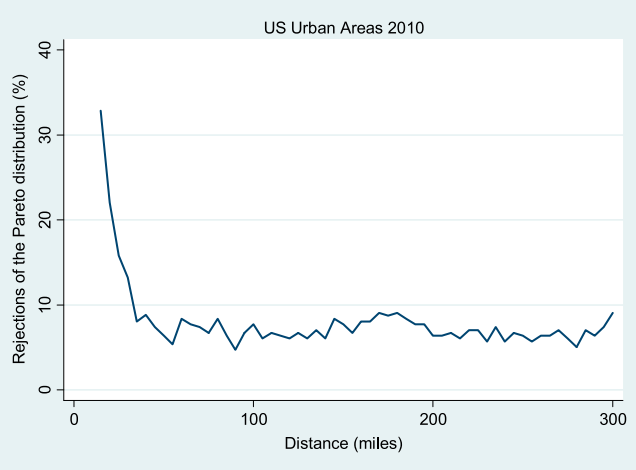

(b) Urban areas

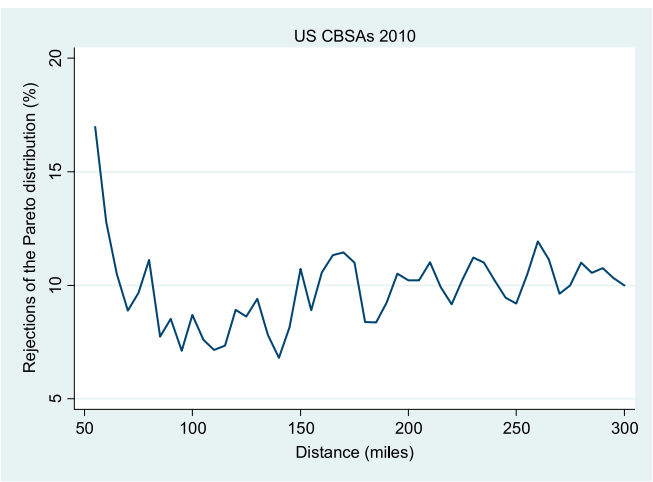

(c) CBSAs

Figure 3. Pareto distribution test over space for geographical samples with a large core city (more than 100,000 inhabitants).

Note: The percentage of rejections of the goodness-of-fit test proposed by Clauset et al. (2009) at the $10 \%$ level.

208,336 and 47,379 sample sizes for places, urban areas and CBSAs, respectively. The surface area $\pi r^{2}$ of a circle is a quadratic function of its radius $r$. Therefore, the number of cities asymptotically will be a quadratic function of $r$. As the radius (i.e., distance) increases, the number of cities included in the circles naturally also increases.

It may be that the results are only driven by sample size, especially because the decreasing relationship between the Pareto exponent and sample size is already known (Eeckhout, 2004). To investigate this issue, placebo regressions were run. The author had previously constructed 58 different geographical samples starting from each city (51 for CBSAs). Now, we construct the same number of samples starting from each city. But instead of including nearby cities, we draw exactly the same number of random cities without replacement from the whole city-size distribution, regardless of the physical bilateral distances. Single-city samples are excluded again. Using the Gabaix and Ibragimov (2011) specification (equation 2), we then estimate the Pareto exponent for all these random samples of cities. Note that sample size is the same in random and geographical samples, but they only share one common element: the initial core city. Finally, we compute the difference between the previously estimated Pareto exponent from the geographical samples and the placebo Pareto exponent obtained from random samples. Therefore, for each city we obtain 58 values of the difference between the Pareto exponents estimated using geographical and random samples (51 in the case of CBSAs). ${ }^{19}$ Alternatively, from the sample size 


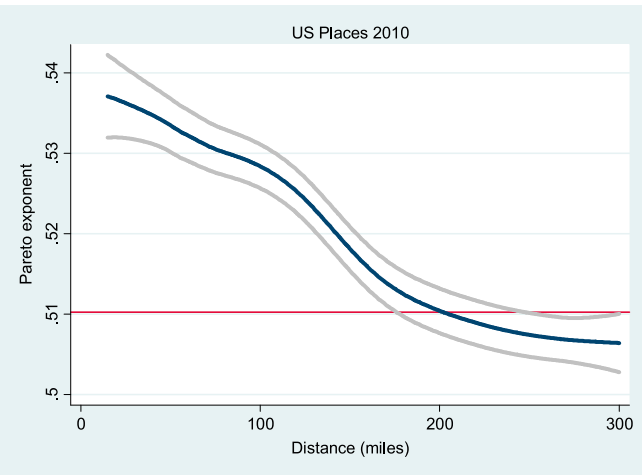

(a) Places

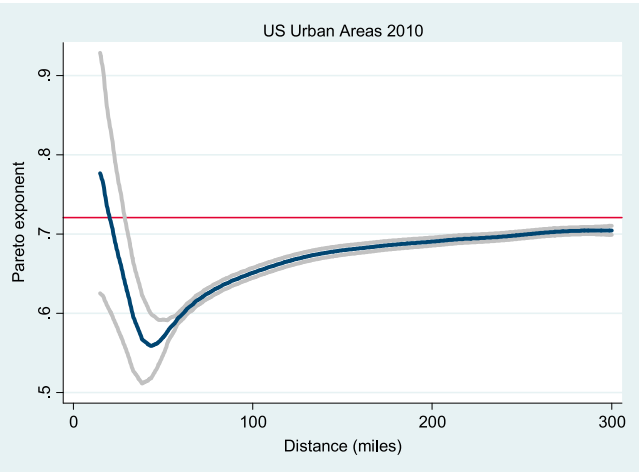

(b) Urban areas

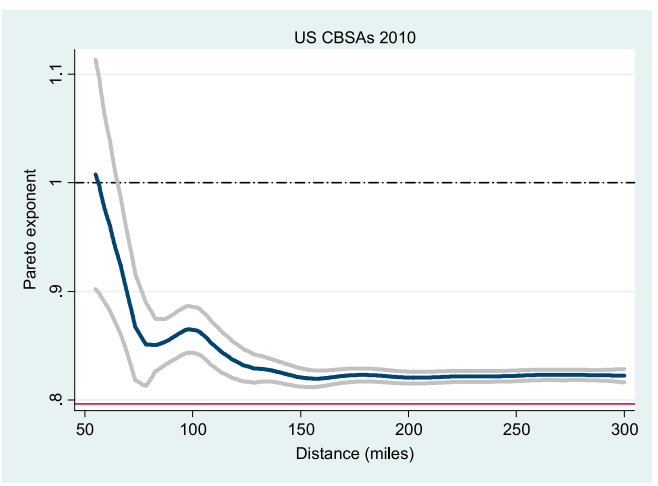

(c) CBSAs

Figure 4. Pareto exponent by distance for geographical samples with a large core city (more than 100,000 inhabitants).

Note: The non-parametric relationship between distance and the estimated Pareto exponents including the 95\% confidence intervals, based on 16,237 (a), 17,034 (b) and 18,530 (c) Pareto exponent-distance pairs. The horizontal line indicates the estimated Pareto exponent for the entire sample of cities.

view, for each number of cities we carry out an average number of 267, 291 and 233 replications in the case of places, urban areas and CBSAs, respectively.

The results are summarized by conducting a non-parametric estimation of the relationship between distance and the difference between the Pareto exponents estimated using geographical and random samples using local polynomial smoothing. Figure 5 shows the results, including the 95\% confidence bands. Note that this time the $x$-axis represents sample size rather than distance. For small sample sizes, the difference between Pareto exponents estimated using geographical and random samples is positive, but decreases with sample size. In the case of urban areas, the difference is not significant for sample sizes smaller than 50 cities. Nevertheless, as sample size increases, the difference stabilizes around a positive value that is significantly different from zero for each of the three city definitions.

The interpretation of a significant positive difference between the Pareto exponents estimated using geographical and random samples is that geography has a significant effect on the value of the Pareto exponent. This effect is not just the consequence of a larger or smaller sample size: Pareto exponents estimated using geographical samples of nearby cities are (on average) higher than those obtained with random samples of cities. This finding indicates that neighbouring cities are more homogeneous in city sizes than random samples of cities. Using data from the United States, 


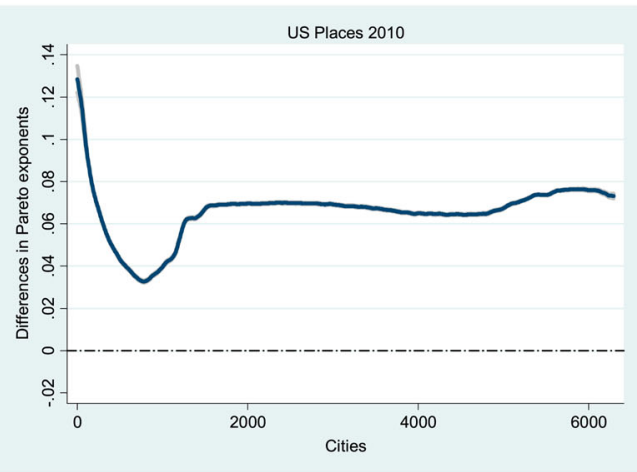

(a) Places

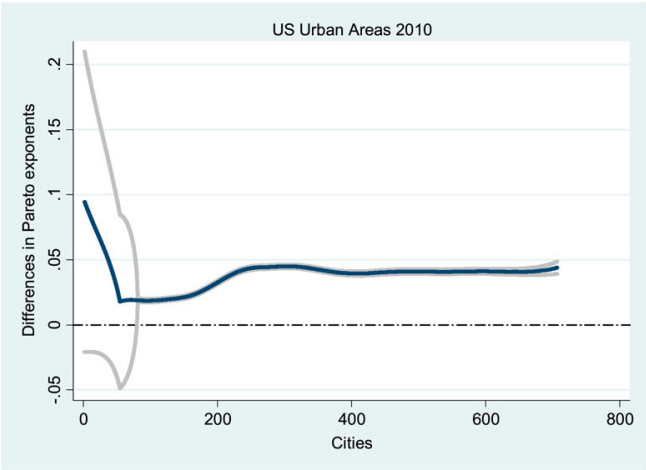

(b) Urban areas

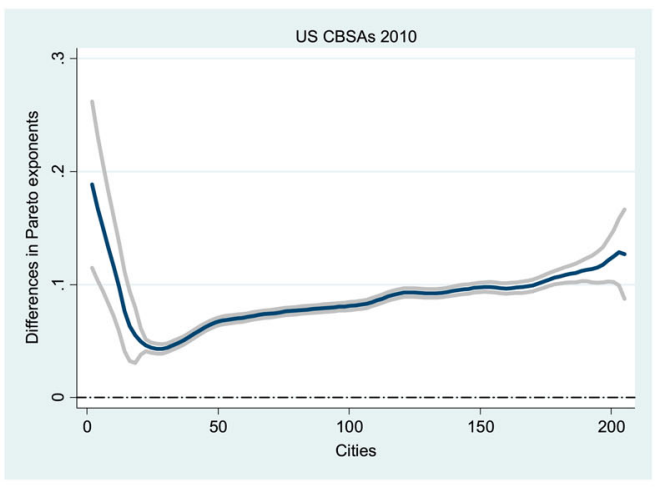

(c) CBSAs

Figure 5. Placebo regressions: differences in Pareto exponents between geographical samples and random samples by sample size.

Note: The non-parametric relationship between distance and the difference between Pareto exponents estimated using geographical and random samples, including the $95 \%$ confidence intervals, based on 1,665,962 (a), 204,959 (b) and 45,912 (c) observations.

Hsu et al. (2014) also find significant differences in the results obtained from spatial partitions of cities and random partitions.

\section{DISCUSSION}

The spatial distribution of population has deep economic and social implications. Economists, statisticians, physicists and geographers have all pointed to the Pareto distribution as a benchmark distribution. In recent years, after an enriching debate, studies from the mainstream literature have been updated to a new paradigm that states that, although most of the city-size distribution is non-linear, the Pareto distribution (and Zipfs law) holds for the largest cities (Giesen et al., 2010; Ioannides \& Skouras, 2013; Levy, 2009).

This paper questions this statement. Large cities are typically far from one another; it is not clear whether we can use theoretical spatial equilibrium models to explain the largest cities as part of an entire city-size distribution, and what it means that the Pareto distribution (and Zipf's law) holds for these largest cities because they are almost independent elements. Rather than focusing on city size, as most studies do, we analyze the validity of the Pareto distribution 
from a spatial perspective, and propose a new distance-based approach. This new methodology enables the following to be confirmed:

- Using all possible combinations of cities within a 300-mile radius, the results indicate that the Pareto distribution cannot be rejected in most cases, regardless of city size and city definition. Therefore, the Pareto distribution fits the city-size distribution well for cities of all sizes as long as they are nearby. Thus, we emphasize that the proper statistical function of city-size distribution is a matter of distance rather than size.

- Eeckhout (2004) concluded that when all US cities are included with no size restriction, citysize distribution is actually lognormal rather than Pareto. This assertion may be right for the whole city-size distribution, ${ }^{20}$ but, as argued above, the city-size distribution for all cities includes elements without any spatial relationship. Therefore, finding support in urban theory for this analysis is difficult. The results show that the Pareto distribution cannot be either discarded or confined to the upper-tail analysis; it is valid for cities of all sizes as long as they are close, which implies that there should be a meaningful spatial relationship between cities (as theoretical models assume).

- Zipfs law only emerges for urban areas and CBSAs at a very particular range of distances (5075 miles for urban areas and 75-80 miles in the case of CBSAs). For the other distances, an exponent with a value of 1 falls outside the confidence bands. Accordingly one can reject Zipfs law for most distances. Regarding places, the estimated Pareto exponents are always less than 1 . Therefore, some evidence supporting Zipf's law can only be found for the aggregate geographical units but not for places that are the lowest spatial unit considered. The literature highlights city definition (Cheshire, 1999; Rosen \& Resnick, 1980; Soo, 2005) as a crucial issue, along with sample size and the choice of the estimator. This paper's spatial perspective adds a new factor influencing the value of the estimated Pareto exponent that has not been considered previously in the literature: distance. Nevertheless, because Zipfs law cannot be rejected for only a small range of distances, the validity of this law may be called into question. Do the results from this spatial approach imply that Zipf s law is a ghost statistic regularity or even that the law has become obsolete? The key point is whether the ranges of distances for which the law is valid have any economic or spatial meaning rather than whether the law holds for a large set of distances. Unfortunately, this question remains open because evidence of the spatial limits of urban systems is not conclusive. Only a few studies have explored this issue (González-Val, 2019a; Hsu et al., 2014) because, as Pumain (2006) points out, systems of cities are difficult to isolate as scientific objects of study.

- Some robustness checks, including placebo regressions, were run and it was shown that there is a significant effect of geography on the Pareto exponent for the three city definitions: Pareto exponents estimated using geographical samples of nearby cities are (on average) higher than those obtained with random samples of cities. This finding indicates that neighbouring cities, which share economic and trade interactions, commuting, and migratory flows, are more homogeneous in city sizes than random samples of cities.

These findings also imply important characteristics for urban hierarchies and the spatial organization of cities. First, the regular hierarchical differentiation of urban systems is typically summarized by a Pareto-like or lognormal distribution of city size (Pumain, 2006); the Pareto distribution suggests complex systems of cities, and the lognormal distribution indicates simple systems of cities. The results support the Pareto distribution for geographical samples of nearby cities, thereby confirming complex systems of cities. According to Chen (2011), this complexity can be external (at the macro-level) and/or internal (at the micro-level).

Second, this type of complex systems of cities involves a hierarchy that is statistically self-similar and hence fractal (Batty, 2006). Therefore, urban systems are a kind of hierarchy with a cascade 
structure, similar to other hierarchies observed in nature, such as the hierarchy of rivers and the energy distributions of earthquakes (Chen \& Zhou, 2008). These hierarchies can be described with a set of exponential laws from which one can derive a set of power laws indicating hierarchical scaling in cities. As Chen (2016) demonstrates, all types of Zipf models can be transformed into the corresponding hierarchies with a cascade structure.

\section{CONCLUSIONS}

This paper uses data from three different definitions of US cities in 2010 (places, urban areas and CBSAs) to introduce a new distance-based approach with the aim of analyzing the influence of distance on the city-size-distribution Pareto exponent using all possible combinations of cities within a 300 -mile radius.

The results lend support to the Pareto distribution, which cannot be rejected in most cases regardless of city size and city definition. The findings have deep implications for urban hierarchies and the spatial organization of cities and raise new questions about the spatial limits of urban systems. These questions, in the author's opinion, deserve more attention from spatial researchers.

\section{ACKNOWLEDGEMENTS}

The assistance of Miriam Marcén with the Stata code is greatly appreciated. The author benefited from the helpful suggestions made by David Cuberes and Fernando Sanz-Gracia. Comments received from two anonymous referees also undoubtedly improved the version originally submitted. All remaining errors are the author's alone. This paper was previously circulated under the title 'City Size Distribution and Space' (Working Papers No. 2017/18), Institut d'Economia de Barcelona (IEB).

\section{DISCLOSURE STATEMENT}

No potential conflict of interest was reported by the author.

\section{FUNDING}

Financial support was provided by the Ministerio de Economía y Competitividad [ ECO201782246-P and ECO2016-75941-R projects], the Gobierno de Aragón (Análisis dinámico espacio-temporal de la realidad económica (ADETRE) research group) and European Regional Development Fund (ERDF).

\section{NOTES}

1 The paper focuses on migrations because it is obvious that there cannot be significant commuting across such wide distances; commuting typically occurs within metropolitan areas from surrounding cities to a central place.

2 Although there are several definitions of cities in the United States, the Census US Gazetteer files only provide coordinates for places, urban areas and CBSAs. Therefore, the use of any other definition of city would imply the use of non-official geographical coordinates.

3 See https://www.census.gov/geo/reference/gtc/gtc_place.html/.

4 See https://www.census.gov/geo/reference/webatlas/uas.html for more information and examples of urban areas.

5 For US Census Bureau definitions for CBSAs, metropolitan and micropolitan statistical, see https://www.census.gov/geo/reference/gtc/gtc_cbsa.html/. 
6 The threshold only indicates the distance at which to stop the procedure, but estimated results for each particular distance are not sensitive to the threshold selection.

7 The haversine formula determines the great circle distance between two points on the surface of the Earth given their longitudes and latitudes, taking into account the mean radius of the Earth. 8 The analysis was repeated by adding 1 mile each time for a few cities; the results were very similar.

9 According to Newman (2006), 'Zipf s law' and 'Pareto distribution' are effectively synonymous with 'power law distribution'.

10 As a robustness check, we also used the statistical test proposed by Gabaix (2009) and Gabaix and Ibragimov (2011) to study the validity of the Pareto distribution; this test is based on a modification of the Rank-1/2 ordinary least squares (OLS) regression. This test has been specifically developed to work with small samples because it reduces the small-sample bias, but the results revealed that the number of rejections of the null of an exact power law significantly increased with the number of cities in the sample. Therefore, the results of Gabaix's (2009) test for urban areas and CBSAs are quite similar to those obtained with Clauset et al.'s (2009) test. However, the results for places using large sample sizes are different because Gabaix's test detects a larger number of rejections of the Pareto distribution than Clauset et al.'s test. These results are available from the author upon request.

11 The procedure is highly computationally intensive. The test was computed with 300 replications for a few cities; the results were similar.

12 Actually, the procedure of Clauset et al. (2009) is specifically designed to select an optimal truncation point. To select the lower bound, the Pareto exponent is estimated for each sample size using the ML estimator, computing the KS statistic for each sample size. The truncation point that is finally selected corresponds to the value of the threshold for which the KS statistic is the smallest. However, in this paper we do not truncate the data. Therefore, the value of the threshold is set to the minimum population in the sample in all cases, considering all the available observations in each geographical sample.

13 The 0.1 reference value for the $p$-value was used, as recommend by Clauset et al. (2009). Other significance levels (1\% and 5\%) yield similar results.

14 By construction, as we start to build up the geographical samples from each city, the number of tests by distance should coincide with the number of cities in the sample. However, in some specific cases with very low sample sizes, the log-likelihood cannot be computed and therefore the test cannot be carried out. Single-city samples are also excluded. Thus, the number of tests by distance is not constant, although the differences are small. The number of tests carried out by distance ranges from 27,886 to 28,755 in the case of places, from 2088 to 3591 for urban areas, and from 796 to 929 for CBSAs.

15 Power laws imply scaling in cities. A power law can often be identified among a certain range of scales, but it must eventually break if the scales of measurements are too large or too small (Bak, 1996; Chen, 2011; Chen \& Zhou, 2008; Williams, 1997). Therefore, it is easier to fit a Pareto distribution to city-size data with a scale-free range compared with using untruncated data.

16 The number of regressions does not coincide exactly with the number of cities multiplied by the 58 different distances considered ( 51 for CBSAs) because in some cases there is only one city in the sample at the start of the procedure with small distances. Therefore, the regression is skipped until there is more than one city in the geographical sample.

17 The lpolyci command in STATA was used with the following options: local mean smoothing, a Gaussian kernel function and a bandwidth determined using Silverman's rule of thumb.

18 Now the number of tests carried out by distance ranges from 276 to 280 in the case of places, from 143 to 298 for urban areas, and from 309 to 370 for CBSAs. 
19 Therefore, the numbers of values is the same than those used previously to obtain Figure 2: 1,665,962 values for places, 204,959 for urban areas and 45,912 for CBSAs.

20 Although some authors find that other non-linear distributions fit city-size data better than a lognormal distribution (Reed, 2002; Giesen et al., 2010; González-Val et al., 2015; Ioannides \& Skouras, 2013; Giesen \& Südekum, 2014).

\section{ORCID}

Rafael González-Val (i) http://orcid.org/0000-0002-2023-5726

\section{REFERENCES}

Auerbach, F. (1913). Das Gesetz Der Bevolkerungskonzentration. Petermanns Geographische Mitteilungen, 49, 7376.

Bak, P. (1996). How nature works: The science of self-organized criticality. New York: Springer.

Batty, M. (2006). N cities and city systems. In D. Pumain (Ed.), Hierarchy in natural and social sciences, Methods Series, Vol. 3 (pp. 143-168). Dordrecht: Springer.

Berry, B. J. L., \& Okulicz-Kozaryn, A. (2012). The city size distribution debate: Resolution for US urban regions and megalopolitan areas. Cities, 29, S17-S23.

Chen, Y. (2011). Modeling fractal structure of city-size distributions using correlation functions. PLoS ONE, 6(9), e24791. doi:10.1371/journal.pone.0024791

Chen, Y. (2016). The evolution of Zipfs law indicative of city development. Physica A: Statistical Mechanics and its Applications, 443, 555-567. doi:10.1016/j.physa.2015.09.083

Chen, Y., \& Zhou, Y. (2008). Scaling laws and indications of self-organized criticality in urban systems. Chaos, Solitons and Fractals, 35(1), 85-98. doi:10.1016/j.chaos.2006.05.018

Cheshire, P. (1999). Trends in sizes and structure of urban areas. In P. Cheshire \& E. S. Mills (Eds.), Handbook of regional and urban economics, Vol. 3 (pp. 1339-1373). Amsterdam: Elsevier.

Clauset, A., Shalizi, C. R., \& Newman, M. E. J. (2009). Power-law distributions in empirical data. SLAM Review, 51(4), 661-703. doi:10.1137/070710111

Cottineau, C. (2017). Metazipf. A dynamic meta-analysis of city size distributions. PLoS ONE, 12(8), e0183919. doi:10.1371/journal.pone.0183919

Dobkins, L. H., \& Ioannides, Y. M. (2001). Spatial interactions among U.S. cities: 1900-1990. Regional Science and Urban Economics, 31, 701-731. doi:10.1016/S0166-0462(01)00067-9

Duranton, G. (2007). Urban evolutions: The fast, the slow, and the still. American Economic Review, 97(1), 197221. doi:10.1257/aer.97.1.197

Eeckhout, J. (2004). Gibrat's law for (all) cities. American Economic Review, 94(5), 1429-1451. doi:10.1257/ 0002828043052303

Fujita, M., Krugman, P., \& Mori, T. (1999). On the evolution of hierarchical urban systems. European Economic Review, 43, 209-251. doi:10.1016/S0014-2921(98)00066-X

Gabaix, X. (1999). Zipfs law for cities: An explanation. Quarterly Journal of Economics, 114(3), 739-767. doi:10. 1162/003355399556133

Gabaix, X. (2009). Power laws in economics and finance. Annual Review of Economics, 1, 255-294. doi:10.1146/ annurev.economics.050708.142940

Gabaix, X., \& Ibragimov, R. (2011). Rank-1/2: A simple way to improve the OLS estimation of tail exponents. Journal of Business and Economic Statistics, 29(1), 24-39. doi:10.1198/jbes.2009.06157

Gabaix, X., \& Ioannides, Y. M. (2004). The evolution of city size distributions. In J. V. Henderson \& J. F. Thisse (Eds.), Handbook of urban and regional economics, Vol. 4 (pp. 2341-2378). Amsterdam: Elsevier.

Garmestani, A. S., Allen, C. R., \& Bessey, K. M. (2005). Time-series analysis of clusters in city size distributions. Urban Studies, 42(9), 1507-1515. doi:10.1080/00420980500185314 
Garmestani, A. S., Allen, C. R., \& Gallagher, C. M. (2008). Power laws, discontinuities and regional city size distributions. Journal of Economic Behavior and Organization, 68, 209-216. doi:10.1016/j.jebo.2008.03.011

Giesen, K., \& Südekum, J. (2011). Zipf's law for cities in the regions and the country. Journal of Economic Geography, 11(4), 667-686. doi:10.1093/jeg/lbq019

Giesen, K., \& Südekum, J. (2014). City age and city size. European Economic Review, 71, 193-208. doi:10.1016/j. euroecorev.2014.07.006

Giesen, K., Zimmermann, A., \& Suedekum, J. (2010). The size distribution across all cities - Double Pareto lognormal strikes. Journal of Urban Economics, 68, 129-137. doi:10.1016/j.jue.2010.03.007

González-Val, R. (2010). The evolution of U.S. city size distribution from a long-term perspective (1900-2000). Journal of Regional Science, 5O(5), 952-972. doi:10.1111/j.1467-9787.2010.00685.x

González-Val, R. (2012). Zipf's law: Main issues in empirical work. Région et Développement, 36, 147-164.

González-Val, R. (2017). City size distribution and space (Working Papers 2017/18). Barcelona: Institut d'Economia de Barcelona (IEB).

González-Val, R. (2019a). The spatial distribution of US cities. Cities. doi:10.1016/j.cities.2018.11.015

González-Val, R. (2019b). Historical urban growth in Europe (1300-1800). Papers in Regional Science. doi:10. 1111/pirs. 12365

González-Val, R., Ramos, A., Sanz-Gracia, F., \& Vera-Cabello, M. (2015). Size distributions for all cities: Which one is best? Papers in Regional Science, 94(1), 177-196.

Hsu, W.-T. (2012). Central place theory and city size distribution. Economic Journal, 122, 903-932. doi:10.1111/j. 1468-0297.2012.02518.x

Hsu, W.-T., Mori, T., \& Smith, T. E. (2014). Spatial patterns and size distributions of cities (Discussion paper No. 882). Kyoto: Institute of Economic Research, Kyoto University.

Ioannides, Y. M., \& Overman, H. G. (2004). Spatial evolution of the US urban system. Journal of Economic Geography, 4(2), 131-156. doi:10.1093/jeg/4.2.131

Ioannides, Y. M., \& Skouras, S. (2013). US city size distribution: Robustly Pareto, but only in the tail. Journal of Urban Economics, 73, 18-29. doi:10.1016/j.jue.2012.06.005

Krugman, P. (1996a). Confronting the mystery of urban hierarchy. Journal of the Japanese and International Economies, 10, 399-418. doi:10.1006/jjie.1996.0023

Krugman, P. (1996b). The self-organizing economy. Cambridge: Blackwell.

Lalanne, A. (2014). Zipfs law and Canadian urban growth. Urban Studies, 51(8), 1725-1740. doi:10.1177/ 0042098013498623

Le Gallo, J., \& Chasco, C. (2008). Spatial analysis of urban growth in Spain, 1900-2001. Empirical Economics, 34, 59-80. doi:10.1007/s00181-007-0150-5

Levy, M. (2009). Gibrat's law for (all) cities: Comment. American Economic Review, 99(4), 1672-1675. doi:10. 1257/aer.99.4.1672

Luckstead, J., \& Devadoss, S. (2017). Pareto tails and lognormal body of US cities size distribution. Physica A: Statistical Mechanics and its Applications, 465, 573-578. doi:10.1016/j.physa.2016.08.061

Malacarne, L. C., Mendes, R. S., \& Lenzi, E. K. (2001). Q-exponential distribution in urban agglomeration. Physical Review E, 65, 017106. doi:10.1103/PhysRevE.65.017106

Newman, M. E. J. (2006). Power laws, Pareto distributions and Zipfs law. Contemporary Physics, 46, 323-351. doi:10.1080/00107510500052444

Nitsch, V. (2005). Zipf zipped. Journal of Urban Economics, 57, 86-100. doi:10.1016/j.jue.2004.09.002

Pisarenko, V. F. (1998). Non-linear growth of cumulative flood losses with time. Hydrological Processes, 12(3), 461470. doi:10.1002/(SICI)1099-1085(19980315)12:3<461::AID-HYP584>3.0.CO;2-L

Pumain, D. (2006). Alternative explanations of hierarchical differentiation in urban systems. In D. Pumain (Ed.), Hierarchy in natural and social sciences, Methodos series, Vol. 3 (pp. 169-222). Dordrecht: Springer.

Rauch, F. (2014). Cities as spatial clusters. Journal of Economic Geography, 14(4), 759-773. doi:10.1093/jeg/lbt034

Reed, W. J. (2001). The Pareto, Zipf and other power laws. Economics Letters, 74, 15-19. doi:10.1016/S0165-1765 (01)00524-9 
Reed, W. J. (2002). On the rank-size distribution for human settlements. Journal of Regional Science, 42(1), 1-17. doi:10.1111/1467-9787.00247

Roberts, D. C., \& Turcotte, D. L. (1998). Fractality and self-organized criticality of wars. Fractals, 6(4), 351-357. doi:10.1142/S0218348X98000407

Rosen, K. T., \& Resnick, M. (1980). The size distribution of cities: An examination of the Pareto law and primacy. Journal of Urban Economics, 8, 165-186. doi:10.1016/0094-1190(80)90043-1

Rossi-Hansberg, E., \& Wright, M. L. J. (2007). Urban structure and growth. Review of Economic Studies, 74, 597624. doi:10.1111/j.1467-937X.2007.00432.x

Soo, K. T. (2005). Zipf's law for cities: A cross-country investigation. Regional Science and Urban Economics, 35, 239-263. doi:10.1016/j.regsciurbeco.2004.04.004

Soo, K. T. (2007). Zipfs law and urban growth in Malaysia. Urban Studies, 44(1), 1-14. doi:10.1080/ 00420980601023869

Williams, G. P. (1997). Chaos theory tamed. Washington, DC: Joseph Henry.

Zipf, G. (1949). Human behaviour and the principle of least effort. Cambridge, MA: Addison-Wesley. 